\title{
Indefinite oscillators and black-hole evaporation
}

\author{
Claus Kiefer* \\ Institut für Theoretische Physik, Universität zu Köln, \\ Zülpicher Straße 77, 50937 Köln, Germany. \\ João Marto ${ }^{\dagger}$ and Paulo Vargas Moniz ${ }^{\ddagger \S}$ \\ Departamento de Fisica, Universidade da Beira Interior, \\ Rua Marquês d'Avila e Bolama, 6200 Covilhã, Portugal.
}

\begin{abstract}
We discuss the dynamics of two harmonic oscillators of which one has a negative kinetic term. This model mimics the Hamiltonian in quantum geometrodynamics, which possesses an indefinite kinetic term. We solve for the time evolution in both the uncoupled and coupled case. We use this setting as a toy model for studying some possible aspects of the final stage of black-hole evaporation. We assume that one oscillator mimics the black hole, while the other mimics Hawking radiation. In the uncoupled case, the negative term leads to a squeezing of the quantum state, while in the coupled case, which includes back reaction, we get a strong entangled state between the mimicked black hole and the radiation. We discuss the meaning of this state. We end by analyzing the limits of this model and its relation to more fundamental approaches.
\end{abstract}

\footnotetext{
*kiefer@thp.uni-koeln.de

†jmarto@ubi.pt

${ }^{\ddagger}$ pmoniz@ubi.pt

§URL: http://www.dfis.ubi.pt/ pmoniz

ฯAlso at CENTRA, IST, Rua Rovisco Pais, 1049 Lisboa Codex, Portugal.
} 


\section{Introduction}

In spite of many attempts, a final theory of quantum gravity remains elusive 11. Such a theory is, however, needed for at least two reasons. First, there are conceptual and formal arguments which point to an encompassing fundamental framework. And second, such a theory is required in order to tackle concrete physical problems. Among the most important ones are problems in cosmology and black-hole physics. In our paper we address the latter field. There, two main issues are usually studied in this context: the microscopic interpretation of black-hole entropy and the description of the final evaporation phase. The first problem is already relevant for large black holes, that is, for black holes with a mass much bigger than the Planck mass. Therefore, some progress has been achieved in various approaches such as string theory, loop quantum gravity, and quantum geometrodynamics [1, 2, 3. On the other hand, as the black hole approaches the Planck mass, a full understanding of the theory is necessary for the final evaporation phase, and it is therefore not surprising that no definitive conclusion has been reached up to now.

In our paper we attempt to shed some light on the question of how the final phase in the black-hole evolution may be described in terms of quantum states used in canonical quantum gravity. In this approach, the central kinematical entities are states that depend - in the gravitational sector - on the three-metric (in quantum geometrodynamics), a non-abelian connection (in quantum connection dynamics), or a SU(2)-holonomy (in loop quantum gravity) [1, 2]. The central 'dynamical' equations are the Hamiltonian and the diffeomorphism constraints (plus, in the latter two versions, the Gauss constraint). The final stage of black-hole evaporation has not yet been described in this language. There exist several attempts using the semiclassical Einstein equations, $G_{\mu \nu}=8 \pi G\left\langle T_{\mu \nu}\right\rangle$, in order to implement the back reaction of the Hawking radiation on the evaporating black hole, with no final result. Since the semiclassical Einstein equations cannot be fundamentally correct [1], a definite answer can only be obtained from the exact theory. It is the purpose of this paper to provide a possible insight into the quantum states of the last evaporation stage. We are certainly not able to present a realistic description of the final stage. But we can address and tentatively answer the following question: Suppose we have solved for the quantum state - how can we recognize black hole evaporation from it? We achieve this goal through some admittedly oversimplified models which, however, as we hope, would capture some real features of the physical process under investigation. The model is mainly chosen in order to study the influence of an indefinite kinetic term, as it occurs in the Wheeler-DeWitt equation. Thus, in this respect our model may exhibit realistic features of the black-hole evaporation process, while in other respects it may be totally unrealistic and must be replaced with features from more realistic (at the moment not exactly soluble) models. We herein envisage the black hole as being embedded as a quantum object into a semiclassical universe. We thus have to address the time-dependent Schrödinger equation with the full quantum gravitational black hole Hamiltonian mimicking the black hole.

Our paper is organized as follows. Section 2 presents a brief review of the semiclassical expansion. We present our arguments why we use a (functional) Schrödinger equation with a quantum gravitational Hamiltonian aiming to describe a quantum black hole. Section 3 is the main part of our paper. We present our model of coupled oscillators mimicking the quantum black hole and Hawking radiation. We first consider the case without direct back reaction (only indirectly through the decrease of the black-hole mass). The negative kinetic term (characteristic of a blackhole Hamiltonian) leads to a squeezed state when the mass which we associate with a black hole approaches the Planck mass. We then discuss the case of a direct coupling. This leads to an entangled state between the mimicked black hole and the Hawking radiation. We calculate and discuss the corresponding reduced density matrices. Section 4 contains our conclusions.

\section{Semiclassical limit and beyond}

The central equations of canonical quantum gravity in the geometrodynamical, connection, or loop approach are of the constraint form $H \Psi=0$. We shall restrict in our paper attention to quantum 
geometrodynamics; from loop quantum gravity one would expect in addition some features arising from the discreteness of space, which are not captured here, but which could play a crucial role in a more realistic scenario [2]. The first point to notice is that the constraint equations in the form $H \Psi=0$ are not yet suitable to describe black-hole evaporation. This is because these equations, as they stand, describe a closed system where everything is described quantum gravitationally. In order words, they would describe the case of a quantum black hole within a quantum universe. Although this may be the appropriate picture at the most fundamental level, the situation that one wants to address is a quantum black hole embedded in a semiclassical universe for which an appropriate time parameter is present.

Such a time variable can arise in various ways. One can, for example, discuss the gravitational collapse of a dust cloud, in which a dust proper time emerges in a natural way, cf. [4] and the references therein. Solutions of quantum geometrodynamics can be exploited to derive Hawking radiation and greybody corrections within this model [5]. Another way of recovering time is a Born-Oppenheimer type of expansion scheme at the full (formal) level of quantum geometrodynamics [1]. This is the framework which we shall use here.

Performing, for example, an expansion with respect to the Planck mass, one can derive from the full constraint equation (the Wheeler-DeWitt equation) a functional Schrödinger equation for 'matter' fields in an external spacetime. The time in this Schrödinger equation is of a semiclassical nature and defined by configurations of the semiclassical gravitational field. More concretely, this equation reads

$$
\begin{aligned}
\mathrm{i} \hbar \frac{\partial}{\partial t}|\psi(t)\rangle & =\hat{H}^{\mathrm{m}}|\psi(t)\rangle \\
\hat{H}^{\mathrm{m}} & \equiv \int \mathrm{d}^{3} x\left\{N(\mathbf{x}) \hat{\mathcal{H}}_{\perp}^{\mathrm{m}}(\mathbf{x})+N^{a}(\mathbf{x}) \hat{\mathcal{H}}_{a}^{\mathrm{m}}(\mathbf{x})\right\}
\end{aligned}
$$

Usually, $\hat{H}^{\mathrm{m}}$ is the Hamiltonian for the 'matter' (i.e. non-gravitational) fields in the Schrödinger picture, parametrically depending on (generally non-static) metric coefficients of the curved spacetime background. The 'bra' and the 'ket' in the quantum state refer to the Hilbert space of the 'matter' fields. ( $N$ and $N^{a}$ are lapse and shift function, respectively, and $\hat{\mathcal{H}}_{\perp}^{\mathrm{m}}(\mathbf{x})$ and $\hat{\mathcal{H}}_{a}^{\mathrm{m}}(\mathbf{x})$ denote the quantum 'matter' part of the Hamiltonian constraint and diffeomorphism constraint operator, respectively.) It is important to emphasize that only the semiclassical degrees of freedom of the gravitational field enter the definition of $t$.

In realistic situations, the full set of these semiclassical variables may, however, include only part of the gravitational field and may also include part of the matter. On the other hand, the gravitons, which are small excitations of the metric, behave fully quantum and must be included into $\hat{H}^{\mathrm{m}}$. But this is also what happens in our case here: The degrees of freedom corresponding to the quantum black hole must be part of $\hat{H}^{\mathrm{m}}$, while the time parameter $t$ is defined by the macroscopic part of gravity and matter, that is, by the semiclassical Universe into which the quantum black hole is embedded.

To describe black-hole evaporation, therefore, one should stick to this semiclassical description of the embedding universe - providing the time parameter $t-$, while employing a full quantum description for the black hole. This corresponds to the realistic situation of an observer residing outside the quantum black hole and having the usual semiclassical time at his disposal. In such a situation one has therefore to apply Equation (1) with $t$ referring to the semiclassical time of the outside universe, and $\hat{H}^{\mathrm{m}}$ being the full Hamilton operator of the quantum black hole and the fields interacting with it. It is for this reason that we consider an indefinite kinetic term in $\hat{H}^{\mathrm{m}}$; it is inherited from the Wheeler-DeWitt equation and applicable here because we apply quantum gravity to the black hole. One thus has to employ a mixture of Schrödinger and Wheeler-DeWitt equation 1

The kinetic term of the gravitational part of the quantum Hamilton operator is suppressed by the Planck mass, $m_{\mathrm{P}}$. As long as the black-hole mass is large, this kinetic term should thus

\footnotetext{
${ }^{1}$ The quantum formation of a black hole from spherical domain-wall collapse was studied recently in a related framework in [6].
} 
be irrelevant. One has in this limit the Hawking radiation as the only contribution to (1). The corresponding quantum state was explicitly calculated and discussed in [7. After the black hole approaches the Planck mass in the final evaporation phase, the full quantum Hamiltonian of the black hole becomes relevant. In the full field theoretic framework of [7, this was not considered. We shall instead investigate this question here in a quantum mechanical model that can capture some of the relevant features. In particular, as emphasized above, we have to deal with the important property of the gravitational Hamiltonian possessing an indefinite kinetic term. This is important for the understanding of time in quantum gravity [1, 8, but will also be crucial for the qualitative features of black-hole evaporation.

\section{A simple model of black-hole evaporation}

The full equation (10) is a complicated functional differential equation for a wave functional depending on the three-metric and matter fields (including Hawking radiation). In order to make our analysis tractable, we shall instead consider the following model, which is purely quantum mechanical:

$$
\begin{aligned}
\mathrm{i} \hbar \frac{\partial}{\partial t} \Psi(x, y, z, t)= & \left(\frac{\hbar^{2}}{2 m_{\mathrm{P}}} \frac{\partial^{2}}{\partial x^{2}}-\frac{\hbar^{2}}{2 m_{y}} \frac{\partial^{2}}{\partial y^{2}}-\frac{\hbar^{2}}{2 m_{z}} \frac{\partial^{2}}{\partial z^{2}}+\right. \\
& \left.\frac{m_{\mathrm{P}} \omega_{x}^{2}}{2} x^{2}+\frac{m_{y} \omega_{y}^{2}}{2} y^{2}+\frac{m_{z} \omega_{z}^{2}}{2} z^{2}\right) \Psi(x, y, z, t) .
\end{aligned}
$$

How do we interpret this ansatz? Let us first recall that the time $t$ in this equation is the semiclassical time of (11) coming from the semiclassical degrees of freedom (such as the scale factor and macroscopic matter) of the Universe. Equation (10) contains second functional derivatives with respect to the three-metric describing the black hole. We shall mimic this three-metric by the single variable $x$; we expect $x$ to be the mass, $M$, of a Schwarzschild black hole (more precisely, its Schwarzschild radius $2 G M / c^{2}$ ). The 'wrong' sign of its kinetic term reflects this correspondence to the three-metric. At a stage where the black hole is quite large, we expect that the kinetic term referring to $x$ is negligible. However, for the final phase which we shall discuss in this paper, $M$ will be small. Therefore, such a term will be of relevance, which is why it has been included into (2).

The variable $y$ is supposed to be the analogue to Hawking radiation, with $m_{y}$ corresponding to its energy, and $z$ stands for the remaining degrees of freedom (with $m_{z}$ as a formal mass parameter). We shall consider in the following only the $x$ - and $y$-variables, that is, we shall restrict attention to the black hole and its interaction with Hawking radiation. We assume that at least for large black holes the evolution is stable in the sense that the potential does not become too negative. For simplicity we have chosen harmonic oscillator potentials; this is realistic for the description of the Hawking radiation [7, but definitely oversimplified for the black hole. In (2) we have not yet taken into account the direct back reaction of Hawking radiation ( $y$-part) onto the black hole ( $x$-part), that is, no $x$ - $y$-coupling is included in (2). This back reaction will be implemented in Section 3.3 below.

We admit that the whole ansatz is not fully realistic, but we hope that our results will shed at least some light on the interpretation of black-hole evaporation in quantum gravity: the setting associated with (2) is not that of a standard quantum mechanical description of harmonic oscillators. On the one hand, there is an unusual kinetic term. As we shall see, the main consequences in our analogue to black-hole evaporation come from the negative kinetic term for $x$ and are independent of the details of the potential. On the other hand, the Planck mass appears as a parameter (suggested from the full Wheeler-DeWitt equation, where it appears in front of the derivatives with respect to the three-metric), participating in (2) as a natural 'suppressor'; this is different from the dynamics of the Hawking radiation [7].

Let us be more precise, taking only the $x$ - and $y$-degrees of freedom into account, one arrives 
at a Schrödinger equation that can be solved by a separation ansatz,

$$
\Psi(x, y, t)=\psi_{x}(x, t) \psi_{y}(y, t)
$$

with (absorbing the separation constant into a state redefinition)

$$
\begin{aligned}
\mathrm{i} \hbar \dot{\psi}_{x}(x, t) & =\left(\frac{\hbar^{2}}{2 m_{\mathrm{P}}} \frac{\partial^{2}}{\partial x^{2}}+\frac{m_{\mathrm{P}} \omega_{x}^{2}}{2} x^{2}\right) \psi_{x}(x, t), \\
\mathrm{i} \hbar \dot{\psi}_{y}(y, t) & =\left(-\frac{\hbar^{2}}{2 m_{y}} \frac{\partial^{2}}{\partial y^{2}}+\frac{m_{y} \omega_{y}^{2}}{2} y^{2}\right) \psi_{y}(y, t) .
\end{aligned}
$$

We see from the first of these equations that $\psi_{x}^{*}$ obeys a Schrödinger equation with standard kinetic term, but with the sign of the potential being reversed ('upside-down oscillator'). This will become important in the following discussion. Wave functions describing the Schwarzschild and the Reissner-Nordström black hole have been discussed in quantum geometrodynamics at various places, see for example [1, 9, 10, 11, 12. This part of the total state is mimicked by the $x$-system. Our purpose here is to construct Gaussian wave packets which are solutions of (4) and (5), and which describe the transition from the semiclassical regime to the final evaporation phase. For various relevant aspects of such wave packets in quantum mechanics, cf. [13, 14, 15].

If $\psi_{0}(x, 0)$ denotes an initial state, the evolution for the $x$-part is found from

$$
\int d x^{\prime} G\left(x, x^{\prime} ; t, 0\right) \psi_{0}\left(x^{\prime}, 0\right)=\psi(x, t)
$$

where $G\left(x, x^{\prime} ; t, 0\right)$ denotes the Green function. In the case of the inverted oscillator it reads ([18, Sec. 6.2.1.8):

$$
G\left(x, x^{\prime} ; t, 0\right)=\sqrt{\frac{m_{\mathrm{P}} \omega_{x}}{2 \pi \mathrm{i} \hbar \sinh \left(\omega_{x} t\right)}} \exp \left[\mathrm{i} m_{\mathrm{P}} \omega_{x} \frac{\left(x^{2}+x^{\prime 2}\right) \cosh \left(\omega_{x} t\right)-2 x x^{\prime}}{2 \hbar \sinh \left(\omega_{x} t\right)}\right] .
$$

We shall now investigate the solutions of the Schrödinger equation for various initial states.

\subsection{Squeezed Ground State}

Before we address the more interesting case of an initial coherent state in Sec. 3.2, we take in a first example as an initial state the ground state of the harmonic oscillator,

$$
\psi_{x 0}^{g}\left(x^{\prime}, 0\right)=\left(\frac{m_{\mathrm{P}} \omega_{x}}{\pi \hbar}\right)^{1 / 4} \exp \left(-\frac{m_{\mathrm{P}} \omega_{x}}{2 \hbar} x^{2}\right) .
$$

For an ordinary oscillator, the system would stay in the ground state. Here, however, we find from (6), together with the complex conjugation, cf. (4), the state

$$
\psi_{x}^{g}(x, t)=\left(\frac{m_{\mathrm{P}} \omega_{x}}{\pi \hbar\left(1-\mathrm{i} \sinh 2 \omega_{x} t\right)}\right)^{1 / 4} \exp \left(-\frac{m_{\mathrm{P}} \omega_{x}}{2 \hbar \cosh 2 \omega_{x} t}\left(1+\mathrm{i} \sinh 2 \omega_{x} t\right) x^{2}\right) .
$$

This is, in fact, a squeezed ground state. Comparing with the general form of Gaussian squeezed states, see for example [17, one recognizes that the squeezing angle is $\phi=\pi / 4$, and the squeezing parameter is $r=\omega_{x} t$. The squeezing thus proceeds along the diagonal in phase space and increases linearly with time. Recalling the analogy to the black-hole case, this squeezing is expected to happen in the final evaporation phase when the kinetic term for the $x$-degree of freedom becomes significant. The squeezing vanishes in the formal limit $m_{\mathrm{P}} \rightarrow \infty$. The evolution of the $x$-part of the wave packet is depicted in Figure 1.

Assuming also for the $y$-part the ground state as initial condition, it is obvious that it will remain in this state if the standard harmonic oscillator propagator is used. The full state is thus the product of (9) for the $x$-part with the standard ground state for the $y$-part. 

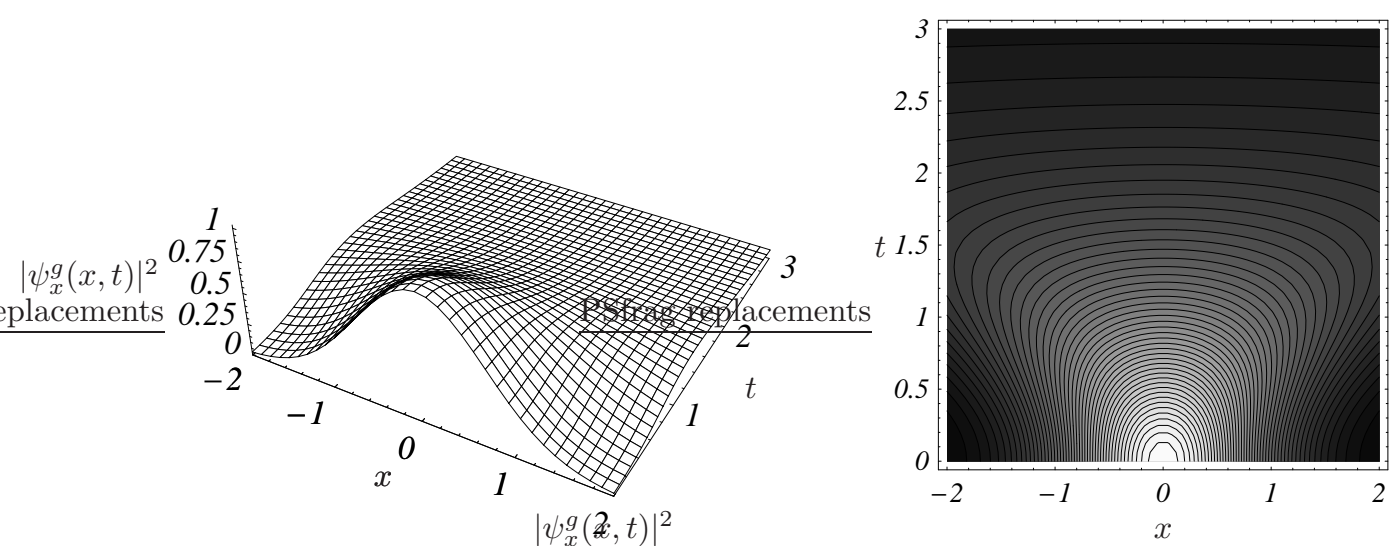

Figure 1: Evolution of a Gaussian state under the inverted oscillator propagator. We depict $\left|\psi_{x}^{g}(x, t)\right|^{2}$ with $m_{\mathrm{P}}=\hbar=\omega_{x}=1$ for simplicity. In the contour plot the brighter areas correspond to higher values for $\left|\psi_{x}^{g}(x, t)\right|^{2}$.

The evaporation of a black hole would thus be mimicked by the squeezing of a Gaussian wave packet. It is known that Hawking radiation is in a squeezed state for semiclassical black holes [18, 19. Here, instead, we have a squeezing of the black-hole quantum state itself.

This case of a squeezed ground state is unrealistic because the support of the wave function in the $x$-variable extends significantly into the negative regime. Therefore we shall turn to a coherent state as an appropriate initial condition.

\subsection{Initial Coherent State}

We shall now consider an initial coherent state for both the $x$ - and the $y$-part. This choice captures more the idea of an initial semiclassical black hole in which the dynamical variable $x$ may be assumed to be concentrated around an initial mass $M$. Addressing first the $x$-part, we have

$$
\psi_{x 0}^{\alpha}(x, 0)=\left(\frac{m_{\mathrm{P}} \omega_{x}}{\pi \hbar}\right)^{1 / 4} \exp \left(-\frac{m_{\mathrm{P}} \omega_{x}}{2 \hbar} x^{2}+\alpha \sqrt{\frac{2 m_{\mathrm{P}} \omega_{x}}{\hbar}} x-\frac{|\alpha|^{2}}{2}-\frac{\alpha^{2}}{2}\right),
$$

where

$$
\alpha=\sqrt{\frac{m_{\mathrm{P}} \omega_{x}}{2 \hbar}} x_{0}+\mathrm{i} \frac{p_{0}}{\sqrt{2 m_{\mathrm{P}} \omega_{x}}},
$$

with $x_{0}$ and $p_{0}$ denoting the expectation values of $x$ and $p_{x}$, respectively. The state (10) thus contains two free parameters $x_{0}$ and $p_{0}$, which together form the complex variable $\alpha$. Application of (7) and complex conjugation then gives

$$
\begin{gathered}
\psi_{x}^{\alpha}(x, t)=\left(\frac{m_{\mathrm{P}} \omega_{x}}{\pi \hbar\left(1-\mathrm{i} \sinh 2 \omega_{x} t\right)}\right)^{1 / 4} \exp \left(-\frac{m_{\mathrm{P}} \omega_{x}}{2 \hbar \cosh 2 \omega_{x} t}\left(1+\mathrm{i} \sinh 2 \omega_{x} t\right) x^{2}\right) \times \\
\exp \left(\mathrm{i} \alpha^{*} \sqrt{\frac{2 m_{\mathrm{P}} \omega_{x}}{\hbar}} \frac{\sinh \omega_{x} t-\mathrm{i} \cosh \omega_{x} t}{\cosh 2 \omega_{x} t} x-\frac{|\alpha|^{2}}{2}-\frac{\alpha^{* 2}}{\cosh 2 \omega_{x} t}\left(\frac{1}{2}+\mathrm{i} \cosh \omega_{x} t\right)\right) .
\end{gathered}
$$

Comparing (12) with (91), we immediately recognize that this state experiences the same degree of squeezing. The state (9) is, of course, a special case of (12) from which it follows for $\alpha=0$. The absolute square of (12) is depicted in Figure 2.

Our model allows both signs for $p_{0}$. In order to capture the idea of an initially decreasing mass we choose as initial condition a negative value for $p_{0}$. This is shown in Figure 2. One can recognize that the localization of the packet centre moves toward smaller values of $x$ before it 
spreads. The spreading in this model should reflect the quantum gravitational behaviour in the realistic situation. In our simplified model it will be possible that also negative values of $x$ can be reached, which is not possible if $x$ represents the Schwarzschild radius; in a more realistic model, a potential wall at $x=0$ should be introduced.


Figure 2: Evolution of $\left|\psi_{x}^{\alpha}(x, t)\right|^{2}$ under the inverted oscillator propagator, where $m_{\mathrm{P}}=\hbar=\omega_{x}=x_{0}=1$ for simplicity and with $p_{0}=-1$. In the contour plot the brighter areas correspond to higher values for $\left|\psi_{x}^{\alpha}(x, t)\right|^{2}$.

The absolute square of (12) can also be written in the form

$$
\begin{aligned}
\left|\psi_{x}^{\alpha}(x, t)\right|^{2}= & \left(\frac{m_{\mathrm{P}} \omega_{x}}{\pi \hbar \cosh 2 \omega_{x} t}\right)^{1 / 2} f\left(x_{0}, p_{0}, t\right) \times \\
& \exp \left(-\frac{m_{\mathrm{P}} \omega_{x}}{\hbar \cosh 2 \omega_{x} t}\left(x-\left[x_{0} \cosh \omega_{x} t+\frac{p_{0} \sinh \omega_{x} t}{m_{\mathrm{P}} \omega_{x}}\right]\right)^{2}\right),
\end{aligned}
$$

where the explicit form of $f\left(x_{0}, p_{0}, t\right)$ is of less interest. It is easily seen from this result that the packet is peaked around the classical solution, but highly squeezed. We note that this fact makes it very sensitive to decoherence, see Section 4.

Taking for the $y$-system (the ordinary oscillator) an initial coherent state as in (10), one obtains the standard result for the time-dependent coherent state, as found in textbooks on quantum mechanics,

$$
\begin{aligned}
\psi_{y}^{\alpha}(y, t)= & \left(\frac{m_{y} \omega_{y}}{\pi \hbar}\right)^{1 / 4} \exp \left(-\frac{\mathrm{i} \omega_{y} t}{2}\right) \exp \left(-\frac{1}{2}\left[\sqrt{\frac{m_{y} \omega_{y}}{\hbar}} y-\sqrt{2} \alpha \mathrm{e}^{-\mathrm{i} \omega_{y} t}\right]^{2}\right) \\
& \times \exp \left(-\frac{1}{2}\left[|\alpha|^{2}-\alpha^{2} \mathrm{e}^{-2 \mathrm{i} \omega_{y} t}\right]\right) .
\end{aligned}
$$

The time evolution of the absolute square is shown in Figure 3. (More realistically, one should take into account a mild squeezing for this state, since it describes Hawking radiation [19].) The total state is then again a product of (12) and (14).

\subsection{Back reaction between a black hole and Hawking radiation}

In the previous section, back reaction was only implicitly implemented in the sense that the kinetic term was assumed to become relevant for small black holes. In order to be more realistic, a direct coupling between the black hole and its Hawking radiation, that is, a coupling between the $x$-and $y$-part, has to be included. This will mimic the process of back reaction more clearly. The simplest way is to include into (2) a linear coupling of the form $\mu x y$, where the parameter $\mu$ should depend 

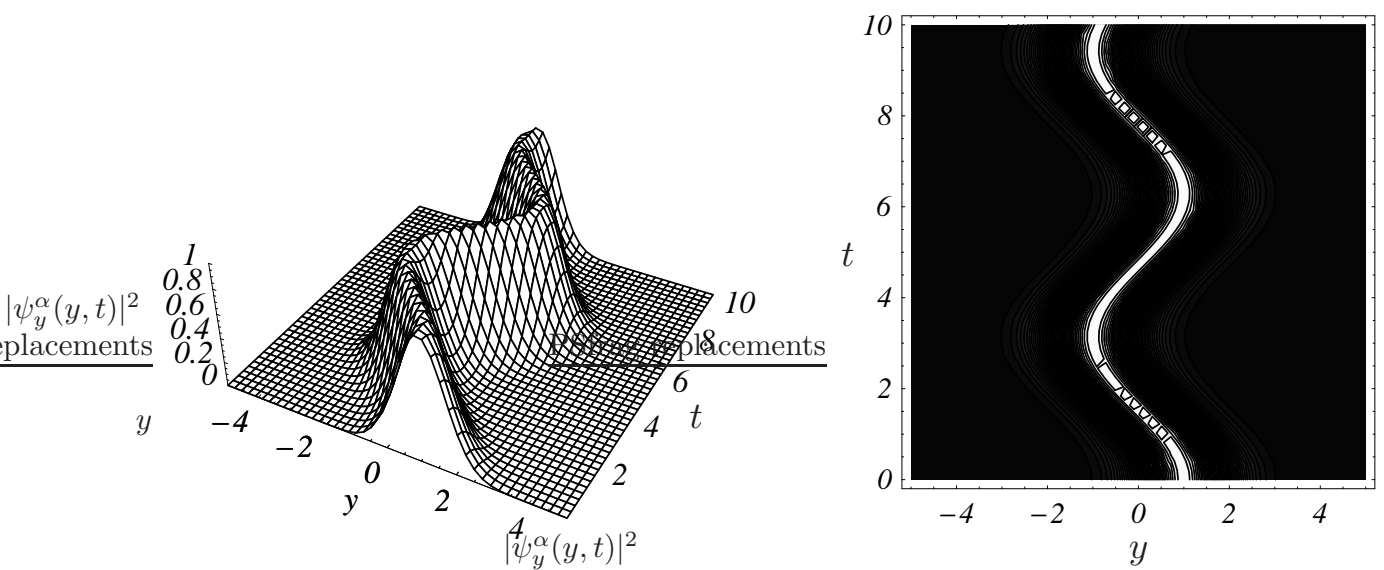

Figure 3: Evolution of $\left|\psi_{y}^{\alpha}(y, t)\right|^{2}$ under the ordinary oscillator propagator, with $m_{y}=\hbar=\omega_{y}=y_{0}=p_{0 y}=1$ for simplicity.

on the black-hole parameters. Then equation (2), when considering only the $x$-and $y$-part, will transform into

$$
\begin{aligned}
\mathrm{i} \hbar \frac{\partial}{\partial t} \Psi(x, y, t)= & \left(\frac{\hbar^{2}}{2 m_{\mathrm{P}}} \frac{\partial^{2}}{\partial x^{2}}-\frac{\hbar^{2}}{2 m_{y}} \frac{\partial^{2}}{\partial y^{2}}+\right. \\
& \left.\frac{m_{\mathrm{P}} \omega_{x}^{2}}{2} x^{2}+\frac{m_{y} \omega_{y}^{2}}{2} y^{2}+\mu x y\right) \Psi(x, y, t) .
\end{aligned}
$$

What would be a suitable initial state? As for the $x$-part, one could still start with an initial coherent state. As for the $y$-part, mimicking Hawking radiation, we would suggest to take the following state as initial state,

$$
\psi_{y 0}^{H}\left(y, t_{0}\right) \propto \exp \left(-\frac{m_{y} \omega_{y}}{2 \hbar} \operatorname{coth}\left[\frac{2 \pi \omega_{y} G M}{c^{3}}+\mathrm{i} \omega_{y} t_{0}\right] y^{2}\right),
$$

where $M$ is the original mass of the (Schwarzschild) black hole, which corresponds to the initial value $x_{0}$ of the $x$-part of the quantum state. What is the justification for this choice? As was shown in [7], (16) corresponds to the state describing Hawking radiation. In [7] we have dealt with a dilaton model; for the Schwarzschild case one would expect the form (16) of the state, cf. [19].

It is appropriate to rewrite Equation (15) in the following way:

$$
\mathrm{i} \hbar \frac{\partial}{\partial t} \Psi\left(Q_{1}, Q_{2}, t\right)=\left[\frac{1}{2}\left(P_{2}^{2}-P_{1}^{2}\right)+\frac{1}{2}\left(\Omega_{1}^{2} Q_{1}^{2}+\Omega_{2}^{2} Q_{2}^{2}\right)+\Gamma Q_{1} Q_{2}\right] \Psi\left(Q_{1}, Q_{2}, t\right),
$$

in which the following redefinitions are used:

$$
\begin{aligned}
x & =\frac{Q_{1} \cos \theta+Q_{2} \sin \theta}{\sqrt{m_{\mathrm{P}}} \cos 2 \theta} & P_{x} & =\sqrt{\frac{m_{\mathrm{P}}}{\cos 2 \theta}}\left(P_{1} \cos \theta+P_{2} \sin \theta\right) \\
y & =\frac{Q_{1} \sin \theta+Q_{2} \cos \theta}{\sqrt{m_{y}} \cos 2 \theta} & P_{y} & =\sqrt{\frac{m_{y}}{\cos 2 \theta}}\left(P_{1} \sin \theta+P_{2} \cos \theta\right) \\
\Omega_{1}^{2} \cos ^{2} 2 \theta & =\omega_{x}^{2} \cos ^{2} \theta+\omega_{y}^{2} \sin ^{2} \theta+\frac{\mu \sin 2 \theta}{\sqrt{m_{\mathrm{P}} m_{y}}} & \widetilde{\omega}_{y} & =\frac{\omega_{y}}{\cos ^{2} 2 \theta} \operatorname{coth}\left[\frac{2 \pi \omega_{y} G M}{c^{3}}+\mathrm{i} \omega_{y} t_{0}\right] \\
\Omega_{2}^{2} \cos ^{2} 2 \theta & =\omega_{x}^{2} \sin ^{2} \theta+\omega_{y}^{2} \cos ^{2} \theta+\frac{\mu \sin 2 \theta}{\sqrt{m_{\mathrm{P}} m_{y}}} & \widetilde{\omega}_{x} & =\frac{\omega_{x}}{\cos ^{2} 2 \theta} .
\end{aligned}
$$


Here, without any loss of generality,

$$
\Gamma=\frac{1}{\cos ^{2} 2 \theta}\left(\frac{1}{2}\left(\omega_{x}^{2}+\omega_{y}^{2}\right) \sin 2 \theta+\frac{\mu}{\sqrt{m_{\mathrm{P}} m_{y}}}\right)
$$

must be zero in order that (17) allows a separation of variables [21]. In this case we find

$$
\mu=-\frac{1}{2} \sqrt{m_{\mathrm{P}} m_{y}}\left(\omega_{x}^{2}+\omega_{y}^{2}\right) \sin 2 \theta
$$

with $\theta \in]-\frac{\pi}{4}, \frac{\pi}{4}$ [ in order to have the set of transformations (18) physically consistent. It is important to point out that $\mu$ can assume any value in the interval $\theta \in]-\frac{\pi}{4}, \frac{\pi}{4}[$.

From (18) and (20) we obtain the following results,

$$
\begin{aligned}
& \Omega_{1}^{2}=\frac{1}{\cos ^{2} 2 \theta}\left[\omega_{x}^{2}\left(\cos ^{2} \theta-\frac{1}{2} \sin ^{2} 2 \theta\right)+\omega_{y}^{2}\left(\sin ^{2} \theta-\frac{1}{2} \sin ^{2} 2 \theta\right)\right] \\
& \Omega_{2}^{2}=\frac{1}{\cos ^{2} 2 \theta}\left[\omega_{x}^{2}\left(\sin ^{2} \theta-\frac{1}{2} \sin ^{2} 2 \theta\right)+\omega_{y}^{2}\left(\cos ^{2} \theta-\frac{1}{2} \sin ^{2} 2 \theta\right)\right] .
\end{aligned}
$$

We are now in a position to apply the same procedures as in Section 3. We must use, of course, the form of the initial states (10) and (16) under the redefinitions (18). In order to obtain the time evolution, the next step consists in calculating the following integral,

$$
\begin{aligned}
\iint d Q_{1}^{\prime} d Q_{2}^{\prime} \quad & G\left(Q_{1}, Q_{1}^{\prime} ; t\right) \cdot G\left(Q_{2}, Q_{2}^{\prime} ; t\right) \\
& \exp \left(-\frac{\widetilde{\omega}_{y}}{2 \hbar}\left(Q_{1}^{\prime 2} \sin ^{2} \theta+Q_{2}^{\prime 2} \cos ^{2} \theta+Q_{1}^{\prime} Q_{2}^{\prime} \sin 2 \theta\right)\right) \\
& \left(\frac{m_{\mathrm{P}} \omega_{x}}{\pi \hbar}\right)^{1 / 4} \exp \left(-\frac{\widetilde{\omega}_{x}}{2 \hbar}\left(Q_{1}^{\prime 2} \cos ^{2} \theta+Q_{2}^{\prime 2} \sin ^{2} \theta+Q_{1}^{\prime} Q_{2}^{\prime} \sin 2 \theta\right)\right. \\
& \left.+\alpha^{*} \sqrt{\frac{2 \widetilde{\omega}_{x}}{\hbar}}\left(Q_{1}^{\prime} \cos \theta+Q_{2}^{\prime} \sin \theta\right)-\frac{|\alpha|^{2}}{2}-\frac{\alpha^{* 2}}{2}\right)
\end{aligned}
$$

where $G\left(Q_{1}, Q_{1}^{\prime} ; t\right)$ is the inverted oscillator propagator and $G\left(Q_{2}, Q_{2}^{\prime} ; t\right)$ the standard one. After some calculation we obtain

$$
\begin{aligned}
\psi\left(Q_{1}, Q_{2}, t\right)=\left(\frac{m_{\mathrm{P}} \omega_{x}}{\pi \hbar}\right)^{1 / 4}\left(-\frac{\Omega_{1} \Omega_{2}}{\mathcal{F}_{1} \mathcal{F}_{3}}\right)^{1 / 2} \exp \left[-\left(\frac{Q_{1}^{2}}{2 \hbar} \frac{\mathcal{F}_{2}}{\mathcal{F}_{1}}+\frac{Q_{2}^{2}}{2 \hbar} \frac{\mathcal{F}_{4}}{\mathcal{F}_{3}}\right)\right] \\
\exp \left[-i \alpha^{*} \sqrt{\frac{2 \widetilde{\omega}_{x}}{\hbar}}\left(\frac{\Omega_{1} Q_{1} \cos \theta}{\mathcal{F}_{1}}+\frac{\Omega_{2} Q_{2} \sin \theta}{\mathcal{F}_{3}}\right)\right] \\
\exp \left[\frac{\Omega_{2} Q_{2} \sin 2 \theta}{2 \mathcal{F}_{1} \mathcal{F}_{3}}\left(\frac{\Omega_{1} Q_{1}}{\hbar}-i \alpha^{*} \sqrt{\frac{2 \widetilde{\omega}_{x}}{\hbar}} \sinh \Omega_{1} t \cos \theta\right)\left(\widetilde{\omega}_{y}+\widetilde{\omega}_{x}\right)\right] \\
\exp \left[-\frac{\hbar \sin ^{2} 2 \theta \sin \Omega_{2} t}{8 \mathcal{F}_{1}^{2} \mathcal{F}_{3}}\left(i \frac{\Omega_{1} Q_{1}}{\hbar}+\alpha^{*} \sqrt{\frac{2 \widetilde{\omega}_{x}}{\hbar}} \sinh \Omega_{1} t \cos \theta\right)^{2}\left(\widetilde{\omega}_{y}+\widetilde{\omega}_{x}\right)^{2}\right] \\
\exp \left[\alpha^{* 2} \widetilde{\omega}_{x}\left(\frac{\cos ^{2} \theta \sinh \Omega_{1} t}{\mathcal{F}_{1}}+\frac{\sin ^{2} \theta \sin \Omega_{2} t}{\mathcal{F}_{3}}\right)-\frac{\alpha^{* 2}}{2}-\frac{|\alpha|^{2}}{2}\right],
\end{aligned}
$$


where we have defined the following functions of time,

$$
\begin{aligned}
\mathcal{F}_{1}= & -i \Omega_{1} \cosh \Omega_{1} t+\widetilde{\omega}_{x} \cos ^{2} \theta \sinh \Omega_{1} t+\widetilde{\omega}_{y} \sin ^{2} \theta \sinh \Omega_{1} t, \\
\mathcal{F}_{2}= & -\Omega_{1}^{2} \sinh \Omega_{1} t-i \Omega_{1} \widetilde{\omega}_{x} \cos ^{2} \theta \cosh \Omega_{1} t-i \Omega_{1} \widetilde{\omega}_{y} \sin ^{2} \theta \cosh \Omega_{1} t, \\
\mathcal{F}_{3}= & -i \Omega_{2} \cos \Omega_{2} t+\widetilde{\omega}_{x} \sin ^{2} \theta \sin \Omega_{2} t+\widetilde{\omega}_{y} \cos ^{2} \theta \sin \Omega_{2} t, \\
& -\left(\widetilde{\omega}_{y}+\widetilde{\omega}_{x}\right)^{2} \sin ^{2} 2 \theta \frac{\sinh \Omega_{1} t \sin \Omega_{2} t}{4 \mathcal{F}_{1}} \\
\mathcal{F}_{4}= & \Omega_{2}^{2} \sin \Omega_{2} t-i \Omega_{2} \widetilde{\omega}_{x} \sin ^{2} \theta \cos \Omega_{2} t-i \Omega_{2} \widetilde{\omega}_{y} \cos ^{2} \theta \cos \Omega_{2} t \\
& +i \Omega_{2}\left(\widetilde{\omega}_{y}+\widetilde{\omega}_{x}\right)^{2} \sin ^{2} 2 \theta \frac{\sinh \Omega_{1} t \cos \Omega_{2} t}{4 \mathcal{F}_{1}} .
\end{aligned}
$$

Evaluating the functions $\mathcal{F}_{n}$ at $t=0$ enables us to recover the initial state $\psi\left(Q_{1}, Q_{2}, t=0\right)$. Now we must perform the inverse coordinate transformation,

$$
\left\{\begin{array}{l}
Q_{1}=\sqrt{m_{\mathrm{P}}} x \cos \theta-\sqrt{m_{y}} y \sin \theta \\
Q_{2}=\sqrt{m_{y}} y \cos \theta-\sqrt{m_{\mathrm{P}}} x \sin \theta
\end{array}\right.
$$

in order to give the explicit form of $\psi(x, y, t)$. Writing

$$
\psi(x, y, t)=F(t) \exp \left(A(t) x^{2}+B(t) x+C(t) y^{2}+D(x, t) y\right),
$$

we find

$$
\begin{aligned}
& F(t)=\left(\frac{m_{\mathrm{P}} \omega_{x}}{\pi \hbar}\right)^{1 / 4}\left(-\frac{\Omega_{1} \Omega_{2}}{\mathcal{F}_{1} \mathcal{F}_{3}}\right)^{1 / 2} \times \\
& \exp \left[\alpha^{* 2} \widetilde{\omega}_{x}\left(\frac{\cos ^{2} \theta \sinh \Omega_{1} t}{\mathcal{F}_{1}}+\frac{\sin ^{2} \theta \sin \Omega_{2} t}{\mathcal{F}_{3}}\right)-\frac{\alpha^{* 2}}{2}-\frac{|\alpha|^{2}}{2}\right] \times \\
& \exp \left[\frac{\alpha^{* 2} \widetilde{\omega}_{x}\left(\widetilde{\omega}_{y}+\widetilde{\omega}_{x}\right)^{2} \cos ^{2} \theta \sin ^{2} 2 \theta \sinh ^{2} \Omega_{1} t \sin \Omega_{2} t}{4 \mathcal{F}_{1}^{2} \mathcal{F}_{3}}\right], \\
& A(t)=-\frac{m_{\mathrm{P}}}{2 \hbar}\left[\frac{\mathcal{F}_{2}}{\mathcal{F}_{1}} \cos ^{2} \theta+\frac{\mathcal{F}_{4}}{\mathcal{F}_{3}} \sin ^{2} \theta+\frac{\Omega_{1} \Omega_{2}}{2 \mathcal{F}_{1} \mathcal{F}_{3}}\left(\widetilde{\omega}_{y}+\widetilde{\omega}_{x}\right) \sin ^{2} 2 \theta\right. \\
& \left.+\frac{\Omega_{1}^{2}\left(\widetilde{\omega}_{y}+\widetilde{\omega}_{x}\right)^{2} \sin ^{2} 2 \theta \cos ^{2} \theta \sin \Omega_{2} t}{4 \mathcal{F}_{1}^{2} \mathcal{F}_{3}}\right], \\
& B(t)=-i \alpha^{*} \sqrt{\frac{2 m_{\mathrm{P}} \widetilde{\omega}_{x}}{\hbar}}\left[\frac{\Omega_{1} \cos ^{2} \theta}{\mathcal{F}_{1}}-\frac{\Omega_{2} \sin 2 \theta}{2 \mathcal{F}_{3}}-\frac{\Omega_{2}\left(\widetilde{\omega}_{y}+\widetilde{\omega}_{x}\right) \sin ^{2} 2 \theta \sinh \Omega_{1} t}{4 \mathcal{F}_{1} \mathcal{F}_{3}}\right. \\
& \left.-\frac{\Omega_{1}\left(\widetilde{\omega}_{y}+\widetilde{\omega}_{x}\right)^{2} \sin ^{2} 2 \theta \cos ^{2} \theta \sinh \Omega_{1} t \sin \Omega_{2} t}{4 \mathcal{F}_{1}^{2} \mathcal{F}_{3}}\right], \\
& C(t)=-\frac{m_{y}}{2 \hbar}\left[\frac{\mathcal{F}_{2}}{\mathcal{F}_{1}} \sin ^{2} \theta+\frac{\mathcal{F}_{4}}{\mathcal{F}_{3}} \cos ^{2} \theta+\frac{\Omega_{1} \Omega_{2}}{2 \mathcal{F}_{1} \mathcal{F}_{3}}\left(\widetilde{\omega}_{y}+\widetilde{\omega}_{x}\right) \sin ^{2} 2 \theta\right. \\
& \left.+\frac{\Omega_{1}^{2}\left(\widetilde{\omega}_{y}+\widetilde{\omega}_{x}\right)^{2} \sin ^{2} 2 \theta \sin ^{2} \theta \sin \Omega_{2} t}{4 \mathcal{F}_{1}^{2} \mathcal{F}_{3}}\right], \\
& D(x, t)=\frac{x \sin 2 \theta}{\hbar} \sqrt{m_{\mathrm{P}} m_{y}}\left(\frac{\Omega_{1} \Omega_{2}}{2 \mathcal{F}_{1} \mathcal{F}_{3}}\left(\widetilde{\omega}_{y}+\widetilde{\omega}_{x}\right) \cos 2 \theta+\frac{\mathcal{F}_{2}}{\mathcal{F}_{1}}+\frac{\mathcal{F}_{4}}{\mathcal{F}_{3}}\right. \\
& \left.+\frac{\Omega_{1}^{2}\left(\widetilde{\omega}_{y}+\widetilde{\omega}_{x}\right)^{2} \sin ^{2} 2 \theta \sin \Omega_{2} t}{8 \mathcal{F}_{1}^{2} \mathcal{F}_{3}}\right) \\
& +\frac{i \alpha^{*}}{2} \sqrt{\frac{2 m_{y} \widetilde{\omega}_{x}}{\hbar}} \sin 2 \theta\left(-\frac{\Omega_{2}}{\mathcal{F}_{1} \mathcal{F}_{3}}\left(\widetilde{\omega}_{y}+\widetilde{\omega}_{x}\right) \cos ^{2} \theta \sinh \Omega_{1} t-\frac{\Omega_{2}}{\mathcal{F}_{3}}+\frac{\Omega_{1}}{\mathcal{F}_{1}}\right. \\
& \left.-\frac{\Omega_{1}\left(\widetilde{\omega}_{y}+\widetilde{\omega}_{x}\right)^{2} \sin ^{2} 2 \theta \sinh \Omega_{1} t \sin \Omega_{2} t}{4 \mathcal{F}_{1}^{2} \mathcal{F}_{3}}\right) .
\end{aligned}
$$


The full state (26) describes an entangled state between this quantum black hole and its Hawking radiation toy model. One can then calculate from it the reduced density matrix for the black hole by integrating out the $y$-part. Tracing out the Hawking radiation thus gives a mixed state for the analogue of the black hole itself [7]. In fact, the presence of entanglement between the black hole state and the Hawking radiation is of particular interest as emphasized, for example, in [20]. It is important to recall that the black hole is an open system, which by itself (without taking into account the Hawking radiation as well as all other fields interacting with it) does not evolve unitarily. The information-loss problem for black holes can only refer to the closed system of black hole plus all other degrees of freedom which are entangled with it. By our very ansatz, in our model the full evolution of black hole plus Hawking radiation is unitary with respect to semiclassical time.

The diagonal element of the reduced density matrix for the black hole is computed from

$$
\rho_{x x}=\operatorname{tr}_{y} \rho=\int|\langle x, y \mid x, y\rangle|^{2} d y,
$$

where we use $|x, y\rangle \equiv \psi(x, y, t)$. Inserting (26) into (28), one finds,

$$
\rho_{x x}=|F|^{2} \exp \left(x^{2}\left(A+A^{*}\right)+x\left(B+B^{*}\right)\right) \sqrt{-\frac{\pi}{C+C^{*}}} \exp \left(\frac{\left(D+D^{*}\right)^{2}}{4\left(C+C^{*}\right)}\right) .
$$

Similarly, the off-diagonal element of the reduced density matrix for the black hole becomes

$$
\rho_{x x^{\prime}}=|F|^{2} \exp \left(x^{2} A+x^{\prime 2} A^{*}+x B+x^{\prime} B^{*}\right) \sqrt{-\frac{\pi}{C+C^{*}}} \exp \left(\frac{\left(D(x)+D^{*}\left(x^{\prime}\right)\right)^{2}}{4\left(C+C^{*}\right)}\right),
$$

where the sum $D(x)+D^{*}\left(x^{\prime}\right)$ implements an additional dependence on $x$ and $x^{\prime}$ as we can verify from (27). Here we shall not discuss further the non-diagonal elements, which describe decoherence effects [7.

Figure 4 depicts $\rho_{x x}$ for $\mu=0$, thus representing a situation where there is no explicit backreaction term. The parameters are specified in order to have $m_{\mathrm{P}} \gg m_{y}$; this seems to be a reasonable choice, since the energy scale of Hawking radiation is much smaller than the Planck mass, except perhaps in the very last stage of the evaporation. We can observe that Figure 4 is essentially identical with Figure 2 (obtained without taking into acount $f\left(x_{0}, p_{0}, t\right)$ ). In fact, having $\mu=0$ corresponds to take $\theta=0$ in (27) and (29); consequently, we obtain the solution (13), since $\lim _{\theta \rightarrow 0} \rho_{x x}=\left|\psi_{x}^{\alpha}(x, t)\right|^{2}$.

The most interesting situation, however, is to allow $\mu \neq 0$ in order to have an idea of the effect of back reaction on $\rho_{x x}$. Figure 5 shows what happens for $\mu$ varying from 0 to 100 . In fact, significant modifications to $\rho_{x x}$ emerge mostly when $\mu$ becomes larger than 1: we can observe a strong modification of $\rho_{x x}$ when the back reaction term comes into play. Due to the entanglement between the black hole state and the Hawking radiation, $\rho_{x x}$ is now sensitive to modifications of $\omega_{y}$ or $m_{y}$. The particular choices of these parameters, which constitute the information carried by Hawking radiation, lead to very different forms of $\rho_{x x}$.

One recognizes from Figure 5 that there is for large $\mu$, that is, for large back reaction, a supression of the strong squeezing. There remains a relatively narrow wave packet whose width, however, strongly oscillates.

Instead of tracing out Hawking radiation from the full entangled state (26), we can trace out the black-hole state and thereby arrive at the density matrix $\rho_{y y}$. The computation of $\rho_{y y}$ processes in a similar fashion as for $\rho_{x x}$, and the formal expression for it is comparable to (29). The interest in this quantity arises from the fact that the effect of the back reaction between the black hole and the Hawking radiation (in the context of this simplified model) can be explored from what effectively leaves the hole. In particular, the issue of information being carried by this radiation is of special interest. The question we may ask is what should we expect to detect if the Hawking radiation were taken to be a signal affected by back reaction effects. In Figure 6 we represent $\rho_{y y}$ for various values of $\mu$. We recognize that the diagrammes for $\rho_{x x}$ and $\rho_{y y}$ look similar for large $\mu$. This could be due to the large entanglement between the black hole and its Hawking radiation, which leads to similar reduced density matrices. 

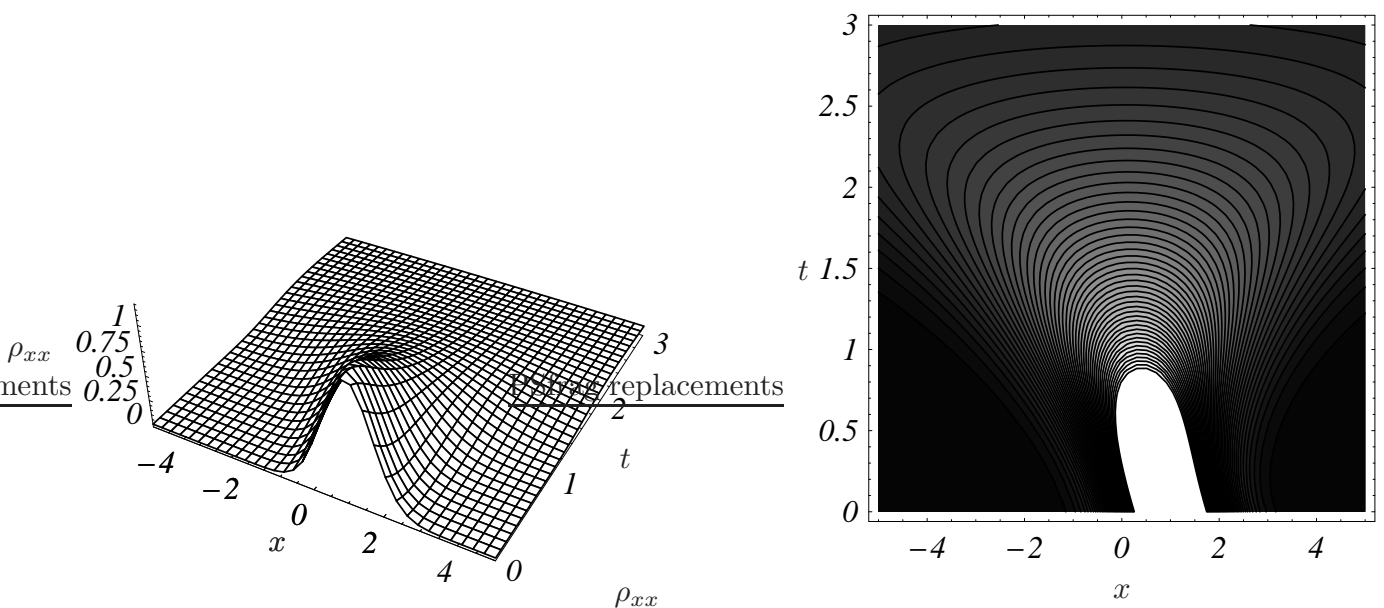

Figure 4: Evolution of $\rho_{x x}$, with $m_{\mathrm{P}}=\hbar=\omega_{x}=x_{0}=1$ and $p_{0}=-1 ; t_{0}=0$ for simplicity, and $\mu=0$ (no back-reaction coupling), $\omega_{y}=\omega_{x} \times 10^{5 / 2}, m_{y}=m_{\mathrm{P}} \times 10^{-5}$. In the contour plot the brighter areas correspond to higher values for $\rho_{x x}$.

\section{Summary and conclusion}

The main purpose of this paper is to get an intuitive, though tentative, insight into how the final phase of black-hole evaporation may look like. In the geometrodynamical framework used here, the question is: suppose we have solved the quantum constraints and found the wave function describing the black hole and its radiation - which characteristics would identify it if some quantum state were detected?

We have argued that in addressing this question we have to deal not with the full quantum constraints of the Universe, but with a (functional) Schrödinger equation which contains the exact Hamiltonian of the quantum black hole together with the semiclassical time $t$ of the rest of the Universe. After all, there are observers who observe the quantum black hole from outside and who have a clock at their disposal.

We have constructed and investigated a very simple model: two coupled harmonic oscillators which mimic black hole and Hawking radiation. We have found that our analogue of a black-hole state experiences a strong squeezing during evaporation, but that this squeezing may disappear for large back reaction; in this limit the reduced density matrices for black hole and Hawking radiation look similar to each other - their differences begin to disappear.

The simplicity of our model allows at best to give some heuristic insight. It is most unlikely that the end phase of a black hole can be described by a system as simple as coupled oscillators. However, it is possible that some of the aspects discussed here - high squeezing of the blackhole state and large entanglement with Hawking radiation - occur also for the realistic quantum states. Moreover, features of an oscillator system are often discussed even in full approaches to quantum gravity, such as the recent proposal for a flux-area operator with an equidistant eigenvalue spectrum [22].

A big open question is the relation of such simplified models like ours to full field-theoretic approaches. This question can, of course, only be answered from the full theory, not from the angle of our model. But there exists an abundance of papers in the literature which address quantum aspects of black holes from this 'minisuperspace' point of view, with $9,10,11,12,23$ listing only some of them. The hope there is always that one or the other result will survive in the full elusive description. Our paper is written in the same spirit as these papers.

Many more questions remain to be answered. One concerns the calculation of the black-hole entropy and the recovery of the Bekenstein-Hawking formula in the limit of large black holes. Great 

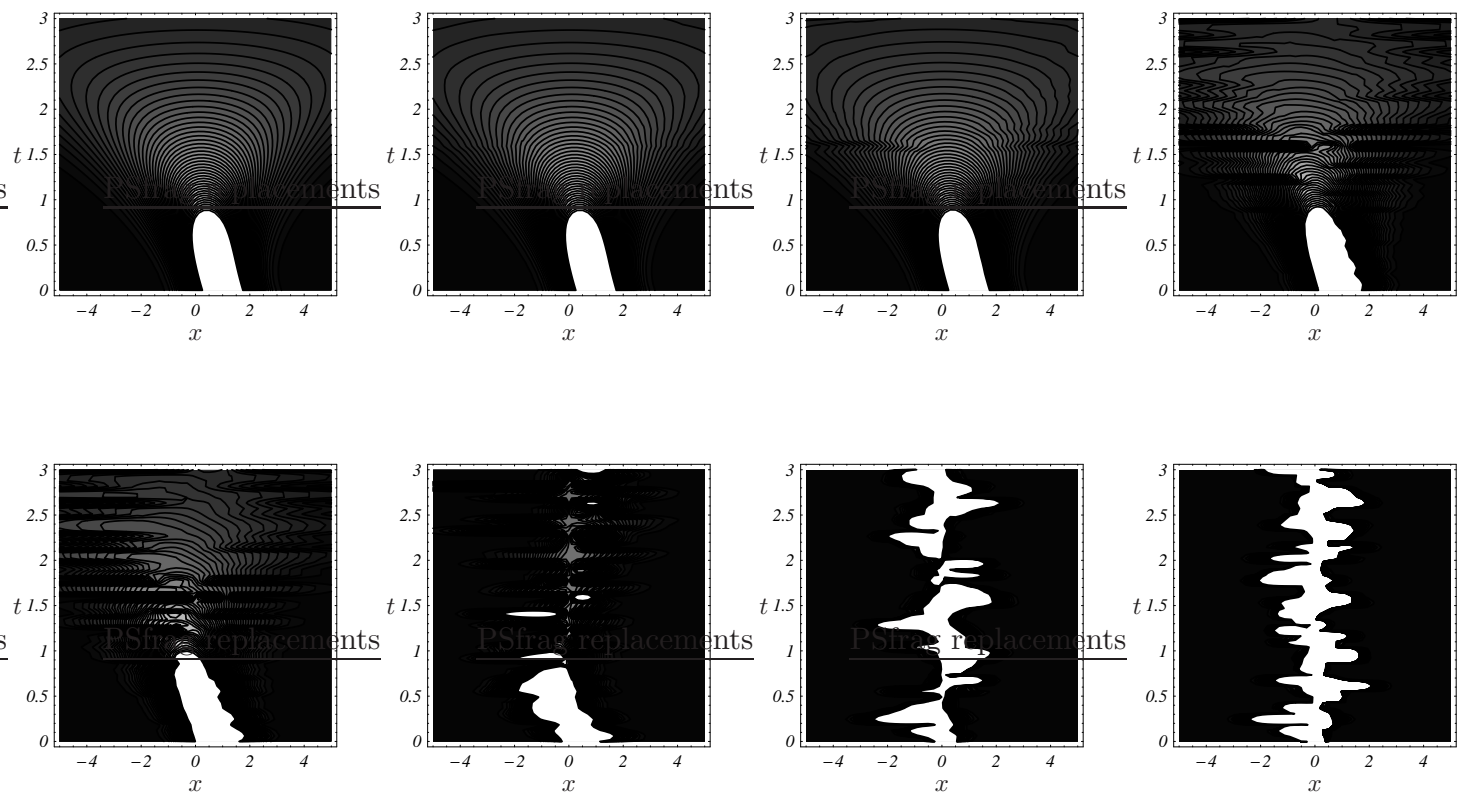

Figure 5: Time evolution of $\rho_{x x}$, with $m_{\mathrm{P}}=\hbar=\omega_{x}=x_{0}=1 ; t_{0}=0$ and $p_{0}=-1$ for simplicity, and $\mu$ (graphics from left to right and top to bottom) assuming the values of the set $\{0,0.5,1,5,10,20,50,100\}, \omega_{y}=\omega_{x} \times 10^{5 / 2}$, $m_{y}=m_{\mathrm{P}} \times 10^{-5}$. In the contour plot the brighter areas correspond to higher values for $\rho_{x x}$.

progress has been achieved here in loop quantum gravity [1, 2] and string theory [3, but some results have also been obtained from the Wheeler-DeWitt equation in quantum geometrodynamics, cf. 24 and the references therein. It would be of interest to give some exact results in the context of our simplified model. One must not forget that a black hole is a genuine open quantum system because it is susceptible to even small interactions with other fields (including its own Hawking radiation, as discussed here) 20 . The process of decoherence would thus play a crucial role in the discussion [25. This is especially important because it is known from quantum mechanics that squeezed states - and the final black-hole state in our model approaches such a state for not too large back reaction - are highly sensitive to decoherence. A possible connection with observation could be made when addressing primordial black holes - small relics from the early Universe, which in the appropriate mass range could be evaporating in the present phase of the Universe [26. We hope to address some of these issues in future publications.

\section{Acknowledgements}

This work was supported by DAAD-GRICES/2004/2005 - D/03/40416, CRUP-AI-A-21/2004, POCI/FIS/57547/2005. C.K. acknowledges kind hospitality at the Universidade da Beira Interior, Covilhã, Portugal, where this work was begun.

\section{References}

[1] C. Kiefer, Quantum Gravity, second edition (Oxford University Press, Oxford, 2007).

[2] C. Rovelli, Quantum Gravity (Cambridge University Press, Cambridge, 2004).

[3] B. Zwiebach, A first course in string theory (Cambridge University Press, Cambridge, 2004). 

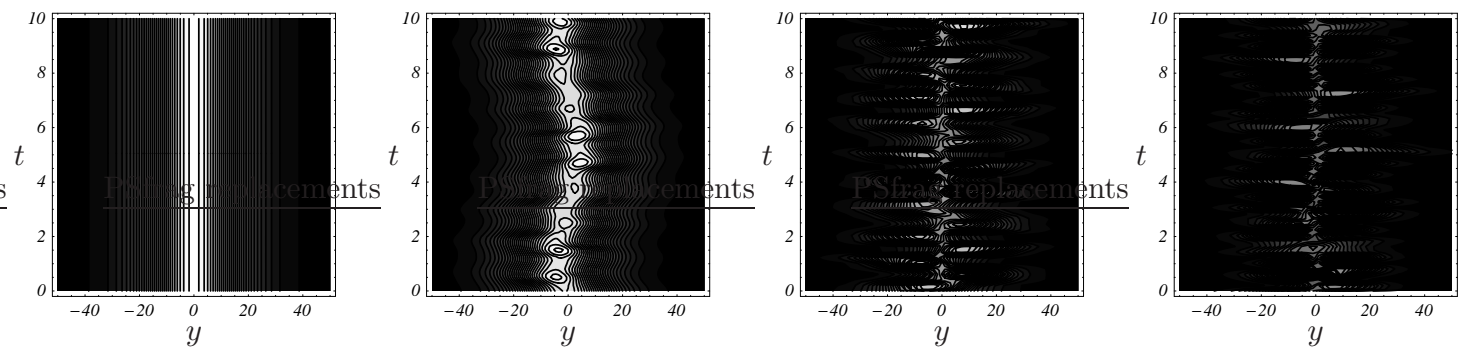

Figure 6: Time evolution of $\rho_{y y}$, with $m_{\mathrm{P}}=\hbar=\omega_{x}=x_{0}=1$ and $p_{0}=-1 ; t_{0}=0$ for simplicity, and $\mu$ (graphics from left to right) assuming the values of the set $\{0,1,5,10\}, \omega_{y}=\omega_{x} \times 10^{5 / 2}, m_{y}=m_{\mathrm{P}} \times 10^{-5}$. In the contour plot the brighter areas correspond to higher values for $\rho_{y y}$. For large values of $\mu$ the results look qualitatively similar to the results for $\rho_{x x}$ depicted in Figure 5. If the back reaction is large, the difference between the black hole and the Hawking radiation begins to disappear.

[4] C. Kiefer, J. Müller-Hill, and C. Vaz, Phys. Rev. D 73, 044025 (2006).

[5] C. Kiefer, J. Müller-Hill, T. P. Singh, and C. Vaz, Phys. Rev. D 75, 124010 (2007); C. Vaz, S. Gutti, C. Kiefer, and T. P. Singh, Phys. Rev. D 76, 124021 (2007).

[6] T. Vachaspati, D. Stojkovic, and L. M. Krauss, Phys. Rev. D 76, 024005 (2007).

[7] J.-G. Demers and C. Kiefer, Phys. Rev. D 53, 7050 (1996).

[8] H. D. Zeh, The physical basis of the direction of time, fifth edition (Springer, Berlin, 2007).

[9] K. V. Kuchař, Phys. Rev. D 50, 3961 (1994).

[10] J. Louko and S. N. Winters-Hilt, Phys. Rev. D 54, 2647 (1996).

[11] C. Kiefer and J. Louko, Ann. Phys. (Leipzig) 8, 67 (1999) gr-qc/9809005.

[12] T. Brotz and C. Kiefer, Phys. Rev. D 55, 2186 (1997).

[13] R. W. Robinett and L. C. Bassett, Found. Phys. Lett. 17, 607 (2004) quant-ph/0408049.

[14] R. W. Robinett and L. C. Bassett, Found. Phys. Lett. 17, 645 (2004) quant-ph/0408050.

[15] M. Andrews, arXiv:0801.0188v1 [quant-ph] (2008).

[16] C. Grosche and F. Steiner, Handbook of Feynman Path Integrals (Springer, Berlin, 1998).

[17] M. N. Nieto, Phys. Lett. A 229, 135 (1997).

[18] L. P. Grishchuk and Y. V. Sidorov, Phys. Rev. D 42, 3413 (1990).

[19] C. Kiefer, Class. Quantum Grav. 18, L151 (2001).

[20] H. D. Zeh, Phys. Lett. A 347, 1 (2005).

[21] F. Benamira and L. Guechi, Czech. J. Phys. 53, 9 (2003) quant-ph/0112003.

[22] J. F. Barbero G., J. Lewandowski, and E. J. S. Villaseñor, arXiv:0905.3465v1 [gr-qc].

[23] H. A. Kastrup and T. Thiemann, Nucl. Phys. B 425, 665 (1994); A. Tomimatsu, Phys. Lett. B 289, 283 (1992); M. Bojowald, Phys. Rev. Lett. 95, 061301 (2005); M. Cavaglia, V. de Alfaro, and A. T. Filippov, Int. J. Mod. Phys. D 5, 227 (1996); J. Mäkelä and P. Repo, Phys. Rev. D 574899 (1998); P. V. Moniz, Mod. Phys. Lett. A 121491 (1997). 
[24] C. Vaz, S. Gutti, C. Kiefer, T. P. Singh, and L. C. R. Wijewardhana, Phys. Rev. D 77, 064021 (2008).

[25] E. Joos, H. D. Zeh, C. Kiefer, D. Giulini, J. Kupsch, and I.-O. Stamatescu, Decoherence and the appearance of a classical world in quantum theory, second edition (Springer, Berlin, 2003).

[26] B. Carr, Lect. Notes Phys. 631, 301 (2003). 